\section{Prescrições de medicamentos para gestantes: um estudo farmacoepidemiológico}

\author{
Drug prescription for pregnant women: \\ a pharmacoepidemiological study
}

\author{
${ }^{1}$ Faculdade de Ciências \\ Metodista de Piracicaba, \\ Piracicaba, Brasil. \\ 2 Faculdade de Saúde \\ Pública, Universidade de \\ São Paulo, São Paulo, Brasil. \\ Correspondência \\ T. A. Carmo \\ Faculdade de Ciências \\ da Saúde, Universidade \\ Metodista de Piracicaba. \\ Rua Nuporanga 20 \\ Piracicaba, $S P$ \\ 13420-252, Brasil. \\ thacarmo@unimep.br
}

\begin{abstract}
Prescription drug use by pregnant women should be viewed as a public health issue, since there are numerous gaps in knowledge on the consequences for both the mother and the fetus. Pharmacoepidemiological studies can help minimize the inherent risks in drug treatment by establishing a profile of drug consumption during pregnancy, providing an evaluation of this service, and identifying intervention measures. The purpose of this study was to establish a prescription profile for pregnant women treated under the National Health System (SUS) in Piracicaba, São Paulo State, Brazil, using prescription indicators recommended by the World Health Organization and the U.S. Food and Drug Administration classification of medicines according to risk to the fetus. According to the current study, during prenatal consultations $44.7 \%$ of the women received drug prescriptions, and the most widely prescribed group of drugs were those acting on the hematopoietic system (34.9\%). Of this total, $26.0 \%$ of the drugs were included in fetal risk category $C, 1.5 \%$ in category $D$, and $1.5 \%$ in category E. These data point to the medicalization of pregnancy and the need for intervention measures aimed at rational prescription drug use during the prenatal period.
\end{abstract}

Drugs; Pregnant Women; Drug Prescriptions
Thais Adriana do Carmo 1 Sandra Maria O. O. Nitrini 2

\section{Introdução}

A utilização de medicamentos por gestantes e seus efeitos sobre o feto passou a ser objeto de grande preocupação após a tragédia da talidomida ocorrida entre 1950 e 1960. Cerca de 10 mil crianças nascidas naquele período apresentaram focomelia, bem como outras alterações congênitas, associadas à utilização deste medicamento durante a gravidez 1 .

Esta catástrofe teve importante repercussão internacional constituindo-se um alerta sobre a questão da segurança na utilização de novos fármacos, da importância de normas mais rigorosas em estudos clínicos antes da liberação de medicamentos para o consumo e a necessidade de criação de órgãos de Farmacovigilância. Especificamente em relação à gravidez, provocou também decisivas mudanças nas atitudes e práticas relativas a prescrição 2,3.

Acreditava-se até então, que a placenta funcionava como uma barreira, protegendo o feto de qualquer agressão farmacológica. Atualmente sabe-se que a maioria dos fármacos contidos nos medicamentos utilizados por gestantes atravessa a placenta e atinge a corrente sangüínea do feto ${ }^{4}$. Deve-se considerar então, que quando uma grávida ingere ou recebe qualquer medicamento dois organismos serão afetados, sendo que um deles (o feto) ainda não tem a mesma capacidade de metabolizar substâncias que a mãe, estando por- 
tanto, mais sujeitos a efeitos negativos não esperados 5 .

Meadows 6 observa que enquanto há certeza do uso e segurança ao feto para alguns medicamentos, na maioria dos casos o conhecimento é limitado, transformando a prescrição medicamentosa na gravidez num grande dilema que deve ser avaliado entre o médico e a paciente. Para a maioria dos medicamentos, $o$ potencial teratogênico é desconhecido 7 .

Um agente pode ser considerado teratogênico quando produz uma alteração, maior ou menor, na morfologia e ou fisiologia normais do feto ${ }^{8}$. Tais alterações, principalmente as má-formações congênitas, têm maior risco de acontecer quando o medicamento com potencial teratogênico é utilizado no primeiro trimestre de gravidez (período de diferenciação embriológica). Nos outros períodos podem ocorrer danos fetais decorrentes de alterações na fisiologia materna, efeitos farmacológicos sobre o feto e interferência no desenvolvimento fetal.

Existem algumas classificações de medicamentos conforme o risco associado ao seu uso durante a gravidez. A classificação adotada pelo Food and Drug Administration (FDA - Estados Unidos) enquadra os medicamentos em cinco categorias 6,7 :

- Categoria A: medicamentos para os quais não foram constatados riscos para o feto em ensaios clínicos cientificamente desenhados e controlados;

- Categoria B: medicamentos para os quais os estudos com animais de laboratório não demonstraram risco fetal (mas não existem estudos adequados em humanos) e medicamentos cujos estudos com animais indicaram algum risco, mas que não foram comprovados em humanos em estudos devidamente controlados;

- Categoria C: medicamentos para os quais os estudos em animais de laboratório revelaram efeitos adversos ao feto, mas não existem estudos adequados em humanos e medicamentos para os quais não existem estudos disponíveis; - Categoria D: medicamentos para os quais a experiência de uso durante a gravidez mostrou associação com o aparecimento de má-formações, mas que a relação risco-benefício pode ser avaliada;

- Categoria X: medicamentos associados com anormalidades fetais em estudos com animais e em humanos e ou cuja relação risco-benefício contra indica seu uso na gravidez.

Outro aspecto que deve ser observado quando se trata da utilização de medicamentos por mulheres grávidas é a provável alteração de características farmacocinéticas dos medicamentos no período da gestação ${ }^{9}$. Segundo dados da literatura internacional, pouco se sabe sobre estas alterações 6,10.

Entretanto, apesar de todos os riscos, o quadro que se apresenta atualmente é o da medicalização da gestante, fenômeno relatado por diferentes autores, tanto em países europeus, Estados Unidos e Brasil 2,3,7,11,12,13, fruto, entre outros determinantes, da lógica de mercado inerente às práticas dos produtores de medicamentos e, por outro lado, da hegemonia da visão mecanicista, biomédica e cartesiana do processo saúde-doença 14 .

\section{Estudos de utilização de medicamentos}

Partindo do pressuposto que não há como abster gestantes da utilização de medicamentos e, conseqüentemente, da exposição de seus filhos aos riscos inerentes a uma terapia medicamentosa, os estudos epidemiológicos, podem ser utilizados para protegê-los de riscos desnecessários 12,15.

A incorporação dos métodos epidemiológicos para estudar e mensurar os eventos medicamento-mediados que ocorrem nas populações, começou a surgir há cerca de trinta anos e a partir daí a Farmacoepidemiologia, ciência constituída por duas grandes áreas: os estudos de eventos adversos ou benéficos decorrentes do uso de medicamentos através de estudos controlados e os Estudos de Utilização de Medicamentos (EUM) 16 .

Os EUM têm como objetivos identificar os sujeitos, os fatores interferentes e o modo como os medicamentos são utilizados nas populações. Tais objetivos são importantes para tomada de decisões não somente em relação aos medicamentos, mas também sobre a cadeia de saúde de forma global, já que o uso de medicamentos se dá dentro de um contexto mais amplo 17 .

A Organização Mundial da Saúde (OMS), baseada nestes conceitos, desenvolveu, padronizou e testou um conjunto de indicadores básicos de utilização de medicamentos publicados em 1993. Tais indicadores são parâmetros altamente normatizados, objetivos, que não precisam ser adaptados a cada país ou a cada realidade e cujo emprego é recomendado para qualquer estudo sobre o uso de medicamentos 18,19.

É importante salientar que cada vez mais os EUM têm sido considerados como importante ferramenta para planejar e gerenciar os servi- 
ços de Assistência Farmacêutica, nortear as políticas de medicamentos e subsidiar as políticas de saúde. Isto porque os medicamentos ocupam atualmente um lugar importante nos sistemas de saúde, tanto no aspecto econômico, quanto nos aspectos técnicos (resolutividade dos serviços) e sociais (uso racional de medicamentos).

Considerando os aspectos apresentados, este trabalho teve como objetivo a investigação das prescrições medicamentosas em gestantes usuárias de um Serviço Público de Saúde, utilizando-se dos indicadores de prescrição preconizados pela OMS, da classificação dos medicamentos prescritos nas categorias de risco ao feto segundo o FDA e de análise dos medicamentos mais prescritos.

\section{Metodologia}

Este trabalho foi desenvolvido no Município de Piracicaba, São Paulo, Brasil, com gestantes usuárias de serviços de pré-natal de atenção primária, no período de janeiro a outubro de 2001. No total foram entrevistadas 427 mulheres (cerca de $5,6 \%$ do total de consultas de prénatal realizadas no período), que faziam o acompanhamento de pré-natal em Unidades Básicas de Saúde (UBS) e na maternidade da Santa Casa de Misericórdia (nesta última somente com gestantes encaminhadas pelo Sistema Único de Saúde - SUS - e matriculadas em UBS sem o serviço de pré-natal).

Tratou-se, segundo Rouquayrol \& Almeida Filho 20, de um estudo individuado - observacional - transversal (ou seccional), baseado em avaliações individuais.

A partir dos objetivos traçados, optou-se, dentre os indicadores propostos pela OMS, por se construir apenas os indicadores de prescrição, entendendo-se que tais indicadores refletem o funcionamento da assistência à saúde em relação a diversos aspectos chaves relacionados à utilização de medicamentos, tais como: grau de polimedicação, tendência de prescrição por nome genérico, abuso de medicamentos específicos (antibióticos e injetáveis) e porcentagem de adesão dos prescritores à lista de medicamentos considerados essenciais pelo município.

Para tentar evitar qualquer viés metodológico, não se entrevistou gestantes de risco ou em atendimento de urgência, mas apenas aquelas em consultas de rotina.

Para definição do tamanho da amostra, partiu-se da hipótese de uma prevalência de $80 \%$ de utilização de pelo menos um medicamento por gestante 2,7,11,12,13 e uma margem de erro tolerável de 5\% (com um nível de confiança de 95\%).

Visando-se garantir também a recomendações da OMS 21 de realização de trinta entrevistas por serviço de saúde, estabeleceu-se que nas unidades onde havia pré-natal seriam envolvidas no mínimo trinta gestantes de cada uma. As demais seriam entrevistadas na maternidade.

Para obtenção dos dados foi utilizado questionário estruturado, aferido em pré-teste realizado após análise e aprovação pelo Comitê de Ética em Pesquisa da Faculdade de Saúde Pública (FSP) da Universidade de São Paulo (USP).

As entrevistas foram realizadas antes e após a consulta médica. Os dados obtidos após a consulta foram destinados à construção dos indicadores de prescrição:

1. Número médio de medicamentos por consulta: média obtida dividindo-se o número total de medicamentos prescritos pelo total de entrevistas realizadas.

2. Porcentagem de medicamentos prescritos por nome genérico (para nome genérico, considerou-se a Denominação Comum Brasileira 22:

$$
\begin{aligned}
& \text { número de medicamentos } \\
& \%=\frac{\text { prescritos pelo nome genérico }}{\text { número total de medicamentos }} \times 100 \\
& \text { receitados }
\end{aligned}
$$

3. Porcentagem de consultas em que se prescreve antibióticos:

$$
\%=\frac{\begin{array}{c}
\text { número de consultas em que } \\
\text { se receita um antibiótico }
\end{array}}{\begin{array}{c}
\text { número total de consultas } \\
\text { envolvidas no estudo }
\end{array}} \times 100
$$

4. Porcentagem de consultas em que se prescreve um medicamento injetável (a única vacina mencionada - dupla adulta - não foi contabilizada como injetável):

$$
\%=\frac{\begin{array}{c}
\text { número de consultas em que } \\
\text { se receita um injetáveis }
\end{array}}{\begin{array}{c}
\text { número total de consultas } \\
\text { envolvidas no estudo }
\end{array}} \times 100
$$

5. Porcentagem de medicamentos prescritos que figuram na lista de medicamentos essenciais (Lista de Medicamentos Padronizados LMP - pelo município):

\footnotetext{
número de medicamentos prescritos mencionados na lista de

$\%=\frac{\text { medicamentos essenciais }}{\text { número total de consultas }} \times 100$ envolvidas no estudo
} 
Partindo-se do pressuposto de que os indicadores do uso de medicamentos são parâmetros de primeiro nível, concebidos, entre outras coisas, para estimular uma análise mais profunda e detalhada em situações específicas e orientar medidas subseqüentes 21 , após identificação dos medicamentos prescritos, buscouse categorizá-los segundo potencial de risco para o feto, utilizando-se a classificação do FDA.

\section{Resultados}

A grande maioria das mulheres envolvidas na pesquisa era de regiões periféricas $(94,1 \%)$ do Município de Piracicaba. A idade variou de 13 a 43 anos, sendo a média de 23,4 anos, com desvio padrão de 5,9 anos e intervalo de confiança entre 5,7 a 41,1 anos. Apenas $25,0 \%$ delas tinham idade acima de 28 anos.

Em relação ao grau de escolaridade, 0,9\% eram analfabetas, $15,7 \%$ estudaram até a 4 a série do Ensino Fundamental e 50,8\% até a 8 a série. Com ensino superior foram encontradas apenas $0,9 \%$ das mulheres.

Considerando-se as 379 respostas obtidas, a renda familiar média foi de 3,4 salários mínimos vigentes (aproximadamente 70 dólares mensais), com desvio padrão de 2,1, sendo que 75,0\% das gestantes tinham renda familiar inferior a 4,4 salários mínimos. A renda per capita por domicílio correspondeu a 0,9 salário mínimo.

Em relação ao trimestre de gestação, 46,7\% das mulheres entrevistadas estavam no último trimestre.

Após serem consultadas, $44,7 \%$ das gestantes tiveram prescrição medicamentosa. O maior número de prescrições medicamentosas ocorreu em gestantes que estavam no segundo trimestre de gestação $(43,5 \%)$.

A Tabela 1 apresenta a classificação dos medicamentos prescritos nas consultas de prénatal segundo os grupos e subgrupos farmacológicos definidos pela Relação Nacional de Medicamentos Essenciais 23 e, dentro de cada grupo, os medicamentos mais freqüentes.

No primeiro trimestre de gestação os grupos farmacológicos mais prescritos foram: medicamentos que atuam sobre o sistema hematopoiético-37,5\%; medicamentos que atuam sobre o sistema digestivo $-26,1 \%$ e os antiinfecciosos $-18,2 \%$.

\section{Indicadores de prescrição (OMS): indicadores de prescrição}

Foram obtidos os seguintes valores:

- Número médio de medicamentos por consulta: 0,6 ;
- Porcentagem de medicamentos prescritos pelo nome genérico: $49,4 \%$;

- Porcentagem de consultas em que foram prescritos antibióticos: 5,6\%;

- Porcentagem de consultas em que foram prescritos medicamentos injetáveis: $0,7 \%$;

- Porcentagem de medicamentos prescritos que constavam na lista de medicamentos padronizados pelo município: $53,5 \%$.

\section{Classificação dos medicamentos prescritos nas categorias de risco ao feto (FDA)}

Na Tabela 2 estão apresentadas as freqüências dos medicamentos prescritos segundo a classificação do FDA, seguida dos três medicamentos mais prescritos dentro de cada uma delas.

\section{Discussão}

Após passarem pela consulta médica de pré-natal, 44,7\% das mulheres receberam prescrição medicamentosa, sendo que o maior número de prescrições $(43,5 \%)$ ocorreu para aquelas que estavam no segundo trimestre de gestação. Nos artigos consultados não foram encontrados parâmetros para inferir sobre a qualificação destes valores. Entretanto, discutir prescrições é aspecto fundamental para buscar uma utilização racional de medicamentos. Segundo a OMS 24, os dados disponíveis mostram que de uma forma geral o pessoal médico e outros profissionais que formulam prescrições estão muito distantes de utilizar os medicamentos racionalmente. Na realidade, a prescrição medicamentosa constitui-se em uma prática definida em um contexto maior e mais complexo, que é o processo saúde - doença como um todo, refletindo as concepções existentes na comunidade na qual está inserida. Acredita-se então, que através da análise das prescrições alguns aspectos interferentes possam ser explicitados e a partir daí proporem-se medidas de intervenção.

De acordo com os dados obtidos, os grupos farmacológicos mais prescritos foram: medicamentos que atuam sobre o sistema hematopoiético-34,9\%; antiinfecciosos - $21,2 \%$; e os que atuam sobre o sistema digestivo $-15,6 \%$. Tais informações são compatíveis com outros artigos consultados. O interessante é que não houve variabilidade destes grupos em função do trimestre de gestação.

Em relação aos medicamentos que atuam sobre o sistema hematopoiético, os produtos tinham como princípio ativo: sulfato ferroso (monodroga), compostos de ferro associados a suplementos vitamínicos, ácido fólico (mono- 
Medicamentos, por nome genérico, prescritos em consultas de pré-natal (SUS) segundo grupo farmacológico (Relação Nacional de Medicamentos Essenciais). Piracicaba, São Paulo, Brasil, 2001.

\begin{tabular}{|c|c|c|c|}
\hline Grupo farmacológico & Medicamento & Freqüência & $\%$ \\
\hline \multicolumn{4}{|l|}{ Analgésicos e medicamentos antienxaquecas } \\
\hline \multirow[t]{2}{*}{ Analgésicos não opióides } & Dipirona* & 1 & 0,4 \\
\hline & Paracetamol & 17 & 6,3 \\
\hline \multicolumn{4}{|l|}{ Antiinflamatórios e antigotosos } \\
\hline \multirow[t]{2}{*}{ Antiinflamatórios não esteróides } & Indometacina & 1 & 0,4 \\
\hline & Diclofenaco de sódio & 1 & 0,4 \\
\hline \multirow[t]{2}{*}{ Antiinflamatórios esteróides } & Triancinolona & 1 & 0,4 \\
\hline & Hidrocortisona* & 1 & 0,4 \\
\hline \multicolumn{4}{|l|}{ Antiinfecciosos } \\
\hline \multirow[t]{8}{*}{ Antibióticos } & Amoxacilina & 1 & 0,4 \\
\hline & Ampicilina & 6 & 2,2 \\
\hline & Benzil penicilina benzatina & 1 & 0,4 \\
\hline & Cefalexina & 14 & 5,2 \\
\hline & Tetraciclina* & 1 & 0,4 \\
\hline & Nitrofurantoína & 3 & 1,1 \\
\hline & Nistatina & 16 & 5,9 \\
\hline & Cetoconazol* & 2 & 0,7 \\
\hline \multirow[t]{3}{*}{ Antifúngicos } & Tinidazol* & 3 & 1,1 \\
\hline & Isoconazol & 2 & 0,7 \\
\hline & Terconazol & 1 & 0,4 \\
\hline \multirow[t]{2}{*}{ Antiparasitários } & Metronidazol & 4 & 1,5 \\
\hline & Espiramicina & 2 & 0,7 \\
\hline Anti-sépticos e desinfetantes & Cetilpiridínio & 1 & 0,4 \\
\hline \multicolumn{4}{|l|}{ Imuno-moduladores e imunoterápicos } \\
\hline Vacina e toxóides & Vacina dupla-adulta & 2 & 0,7 \\
\hline \multicolumn{4}{|l|}{ Nutrientes } \\
\hline Nutrição e reposição hídrica parental & Glicose $25 \%$ & 1 & 0,4 \\
\hline \multirow[t]{5}{*}{ Vitaminas e sais minerais } & Vitamina $A^{*}$ & 7 & 2,6 \\
\hline & Vitamina C & 1 & 0,4 \\
\hline & Vitamina $C^{*}$ & 3 & 1,1 \\
\hline & Cálcio & 1 & 0,4 \\
\hline & Vitamina B6* & 7 & 2,6 \\
\hline \multicolumn{4}{|l|}{$\begin{array}{l}\text { Medicamentos que atuam sobre } \\
\text { o sistema cardiovascular }\end{array}$} \\
\hline Antihipertensivos & Metildopa & 3 & 1,1 \\
\hline \multicolumn{4}{|l|}{ Dermatológicos } \\
\hline \multirow[t]{2}{*}{$\begin{array}{l}\text { Medicamentos antipruriginosos } \\
\text { e antiinflamatórios tópicos }\end{array}$} & Lisozima & 1 & 0,4 \\
\hline & $\begin{array}{l}\text { Ácido mucopolissacárido- } \\
\text { polissulfúrico }\end{array}$ & 1 & 0,4 \\
\hline \multicolumn{4}{|l|}{$\begin{array}{l}\text { Medicamentos que atuam sobre } \\
\text { o sistema respiratório }\end{array}$} \\
\hline \multirow[t]{2}{*}{ Anti-asmáticos } & Fenilefrina* & 2 & 0,7 \\
\hline & Etafedrina* & 1 & 0,4 \\
\hline \multirow{3}{*}{ Antitussígenos e fluidificantes } & Ambroxol & 2 & 0,7 \\
\hline & Bromexina & 2 & 0,7 \\
\hline & lodeto de potássio* & 3 & 1,1 \\
\hline \multicolumn{4}{|l|}{$\begin{array}{l}\text { Medicamentos que atuam sobre o sistema endócrino } \\
\text { reprodutor e produtos farmacêuticos correlatos }\end{array}$} \\
\hline \multirow[t]{2}{*}{ Medicamentos que atuam na motilidade uterina } & Hesperidina* & 8 & 3,0 \\
\hline & Isoxsuprina & 1 & 0,4 \\
\hline
\end{tabular}

(continua) 
Tabela 1 (continuação)

\begin{tabular}{|c|c|c|c|}
\hline Grupo farmacológico & Medicamento & Freqüência & $\%$ \\
\hline \multicolumn{4}{|l|}{$\begin{array}{l}\text { Medicamentos que atuam sobre } \\
\text { o sistema hemocitopoiético }\end{array}$} \\
\hline Antianêmicos & $\begin{array}{l}\text { Ácido fólico } \\
\text { Ácido fólico* } \\
\text { Sulfato ferroso } \\
\text { Ferro* }\end{array}$ & $\begin{array}{r}5 \\
2 \\
60 \\
27\end{array}$ & $\begin{array}{r}1,8 \\
0,7 \\
22,3 \\
10,0\end{array}$ \\
\hline \multicolumn{4}{|l|}{$\begin{array}{l}\text { Medicamentos que atuam sobre } \\
\text { o sistema digestivo }\end{array}$} \\
\hline Antiulcerosos & Cimetidina & 2 & 0,7 \\
\hline Antiácidos & $\begin{array}{l}\text { Hidróxido de Alumínio * } \\
\text { Hidróxido de Alumínio }\end{array}$ & $\begin{array}{r}11 \\
3\end{array}$ & 4,1 \\
\hline Antieméticos e agentes pró-cinéticos & $\begin{array}{l}\text { Metoclopromida } \\
\text { Dimenidrato } \\
\text { Bromoprida }\end{array}$ & $\begin{array}{l}2 \\
2 \\
3\end{array}$ & $\begin{array}{l}0,7 \\
0,7 \\
1,1\end{array}$ \\
\hline \multicolumn{4}{|l|}{ Antidiarréicos sintomáticos } \\
\hline $\begin{array}{l}\text { Antiespasmódicos e correlatos } \\
\text { Laxativos }\end{array}$ & $\begin{array}{l}\text { Hioscina } \\
\text { Bisacodil }\end{array}$ & $\begin{array}{r}18 \\
1\end{array}$ & $\begin{array}{l}6,7 \\
0,4\end{array}$ \\
\hline \multicolumn{4}{|c|}{$\begin{array}{l}\text { Outros (medicamentos que não se enquadram } \\
\text { em nenhum grupo) }\end{array}$} \\
\hline & Dexpantenol & 1 & 0,4 \\
\hline & Dimeticona & 1 & 0,4 \\
\hline & Nafazolina* & 2 & 0,7 \\
\hline & Passiflora* & 1 & 0,4 \\
\hline Total & & 269 & 100,0 \\
\hline
\end{tabular}

* em associação com outras substâncias.

droga) e ácido fólico associado em suplementos vitamínicos.

Quanto ao uso de sais de ferro na gestação, percebe-se se trata de uma intervenção de rotina 2,7. Entretanto, Mengue et al. 7 ressaltam que as evidências que sustentam esta conduta são fracas e os benefícios do tratamento não são claramente definidos.

A favor da prescrição de suplementos de ferro, está a recomendação da OMS de que devido às altas taxas de anemia nos países de terceiro mundo, deve-se ministrar tais medicamentos a todas as mulheres nos últimos quatro a cinco meses de gravidez 25 .

Entretanto, contra a prescrição de suplementos de ferro de maneira generalizada, está o fato de que somente 5,0 a $10,0 \%$ do ferro ingerido é absorvido em condições normais. O organismo humano só absorve ferro em proporções maiores $(25,0 \%)$ em situações de deficiência 9 . Deste modo, a ingestão diária através da dieta é geralmente suficiente para manter os níveis adequados de ferro no organismo, justificandose a utilização de medicamentos com ferro somente em casos de anemia diagnosticada 25 .

Além disto, devido à má absorção do sal, as preparações de ferro freqüentemente podem causar alterações gastrointestinais 25,26 e exis- tem trabalhos sugerindo propriedades teratogênicas quando da sua ingestão no primeiro trimestre de gravidez 25,27.

Considerando como dose profilática 25 a $30 \mathrm{mg}$ de ferro elementar/dia 9,28, vale destacar que nas prescrições analisadas $94,8 \%$ dos medicamentos com ferro estavam em concentrações acima da dose recomendada, embora ainda dentro da faixa terapêutica (180mg/dia). Esta informação é importante porque se sabe que os efeitos adversos do ferro oral são geralmente dose-dependente e a dose tóxica é muito próxima da dose terapêutica ${ }^{9}$. Estes dados sugerem um uso inadequado dos suplementos de ferro, sujeitando as gestantes e seus fetos a efeitos adversos desnecessário.

Em relação ao ácido fólico, vitamina hidrossolúvel do complexo $\mathrm{B}$, sabe-se que a deficiência materna ou a utilização de medicamentos antagonistas do seu metabolismo no período periconcepcional (de um a dois meses antes do último período menstrual) estão associados a um aumento do risco de defeitos no tubo neural (DTN) 29,30.

Nesta perspectiva, Werler et al. 30 descrevem estudo caso-controle, onde os resultados obtidos sugerem que uma suplementação diária de $0,4 \mathrm{mg}$ entre os dois meses antes da con- 
Distribuição dos medicamentos prescritos em consultas de pré-natal (SUS), segundo categorias de risco ao feto (FDA), e os três mais freqüentes (por nome genérico) dentro de cada categoria. Piracicaba, São Paulo, Brasil, 2001.

\begin{tabular}{|c|c|c|c|c|c|}
\hline Categorias & Freqüência & $\%$ & Medicamentos & Freqüência & $\%$ \\
\hline \multirow[t]{3}{*}{ A } & 96 & 35,7 & Sulfato ferroso & 60 & 62,5 \\
\hline & & & Ferro (assoc.) & 27 & 23,1 \\
\hline & & & Vitamina A (assoc.) & 3 & 3,1 \\
\hline \multirow[t]{3}{*}{ B } & 74 & 27,5 & Paracetamol & 17 & 23,9 \\
\hline & & & Nistatina & 16 & 22,5 \\
\hline & & & Cefalexina & 14 & 19,7 \\
\hline \multirow[t]{3}{*}{ C } & 70 & 26,0 & Hioscina & 18 & 25,1 \\
\hline & & & Hidróxido de alumínio (assoc.) & 11 & 15,7 \\
\hline & & & Vitamina B6* & 7 & 10,0 \\
\hline \multirow[t]{3}{*}{$D$} & 5 & 1,5 & lodeto de potássio (assoc.) & 3 & 60,0 \\
\hline & & & Tetraciclina (assoc.) & 1 & 20,0 \\
\hline & & & Indometacina** & 1 & 20,0 \\
\hline E & 4 & 1,5 & Vitamina A (assoc.) (acima de $3.000 \mathrm{UI}$ ) & 4 & 100,0 \\
\hline \multirow[t]{4}{*}{ Sem informações } & 20 & 7,3 & Hesperidina (assoc.) & 8 & 40,0 \\
\hline & & & Bromoprida & 3 & 15,0 \\
\hline & & & Ambroxol & 2 & 10,0 \\
\hline & & & Isoconazol & 2 & 10,0 \\
\hline
\end{tabular}

* classificação dependente da dose;

** quando utilizado no terceiro trimestre de gestação.

cepção e o início da gestação reduz os riscos de má-formação, entre elas a ocorrência de espinha bífida, defeito nos lábios e no palato, máformação do coração, membros, trato urinário e cérebro.

De fato, o relato do uso de ácido fólico por gestantes é comum tanto nos artigos consultados quanto foi no presente trabalho. Entretanto, assim como em relação aos suplementos de ferro, o que chama a atenção é a concentração dos medicamentos utilizados. Considerando como dose recomendada uma suplementação diária de $0,4 \mathrm{mg}$ de ácido fólico no período perinatal e até a décima segunda semana de gravidez 25 , do total de prescrições $47,9 \%$ estavam acima da dose diária recomendada. Vale ressaltar que até a concentração de $0,8 \mathrm{mg} / \mathrm{dia}$, o ácido fólico é considerado categoria A de risco ao feto. Acima disto passa a ser categoria C 31 . Outro aspecto a ser destacado é a prescrição de ácido fólico para mulheres no segundo e no terceiro trimestre de gravidez, quando já não ocorre seu efeito protetor contra má-formação congênita.

Levando-se em consideração que os suplementos de ácido fólico também podem causar efeitos colaterais tais como: choque anafilático, prurido, erupção na pele, estreitamento dos brônquios e mal estar 32 , os dados obtidos sugerem a necessidade de adequação de quase metade das prescrições na busca de minimizar os problemas decorrentes da utilização de tais medicamentos.

Quanto à utilização do sulfato ferroso e do ácido fólico em associação a outras vitaminas, os resultados são bastante controversos. Segundo Chetley 25 , não há justificativa para o uso de tais medicamentos. Por outro lado, Scholl et al. 33 relatam estudo realizado nos Estados Unidos, com mulheres de baixa renda, onde a ingestão de multivitamínicos contribuiu para um ganho de peso adequado das gestantes e uma diminuição de partos prematuros.

Diniz 34 ressalta que a deficiência de Vitamina A é também um dos mais importantes problemas nutricionais dos países em desenvolvimento. E parece estar relacionada com o aparecimento de má-formação congênita. Entretanto, destaca que altas doses de Vitamina A podem ser teratogênicas, principalmente quando administradas no início da gravidez. Por is- 
so a suplementação indiscriminada não é recomendada.

De fato, doses acima de 10 mil UI de Vitamina A diárias são consideradas categoria X de risco ao feto 31 , ou seja, possuem teratogenicidade confirmada e não devem ser usadas na gestação. Berglund et al. 4 citam a associação de má-formação óssea e craniana em crianças a doses excessivas da Vitamina A e seus derivados.

Em relação à classificação dos medicamentos pelo risco ao feto, sugere-se que cerca de $40,0 \%$ dos medicamentos prescritos às gestantes foram de medicamentos cuja segurança de uso na gravidez pode ser questionada, pois coloca em risco a formação das futuras crianças. O mais grave, porém, foi a prescrição de medicamentos sabidamente teratogênicos.

Quanto aos resultados obtidos para os indicadores é importante salientar que, segundo Hogerzeil et al. 35, para três deles é muito difícil definir valores ótimos. São os seguintes: o número médio de medicamentos por consulta, porcentagem de consultas em que se prescrevem antibióticos e porcentagem de consultas em que se prescrevem injetáveis. Isto ocorre porque tais indicadores sofrem influência de diferentes fatores fortemente dependentes de características regionais e locais (perfil de morbidade, características dos serviços de saúde, existência de lista de medicamentos padronizados, entre outros) 36.

Por outro lado, para os indicadores que medem a porcentagem de medicamentos prescritos pelo nome genérico e a porcentagem dos medicamentos prescritos que constam da lista de medicamentos padronizados, os valores são óbvios: eles devem ser o mais alto possível, considerando-se que:

- Se existe uma lista de medicamentos padronizados, de medicamentos essenciais àquela localidade ou serviço, ela deve ser seguida, a menos que não reflita as demandas existentes (nestes casos valores baixos podem indicar necessidade de sua revisão);

- A prescrição por nome genérico faz parte das estratégias da OMS para se aumentar o acesso da população ao medicamento e promover a utilização racional dos mesmos 24 .

Sendo assim, os três indicadores abaixo não podem ser avaliados em relação a sua adequação, mas devem ser analisados dentro de um contexto:

- Número médio de medicamentos por consulta: 0,6 ;

- Porcentagem de consultas em que se prescreveram antibióticos: 5,6\%;

- Porcentagem de consultas em que se prescreveram injetáveis: $0,7 \%$.
A princípio parecem valores baixos se comparados com os relatados em outros trabalhos, entretanto, frente à observação de que cerca de $77,0 \%$ das prescrições avaliadas apresentavam algum tipo de problema (risco potencial ao feto e ou dose ou posologia inadequadas) constituem-se apenas em dados descritivos não devendo ser agregados a nenhum juízo de valor.

Quanto aos demais indicadores (porcentagem de medicamentos prescritos pelo nome genérico e porcentagem de medicamentos prescritos constantes da LMP), pode-se dizer que os valores encontrados são baixos, sugerindo a necessidade de discussão das práticas de prescrição. Primeiro, porque, a partir deles, parece haver desconhecimento da legislação por parte dos prescritores: a metade dos medicamentos prescritos não estava grafada pelo nome genérico. Segundo, porque estes dados revelam a não adesão dos profissionais às políticas nacional e local de medicamentos e conseqüentemente à Política de Saúde. E, por outro lado, a alta prescrição de medicamentos não-padronizados, sugere a necessidade de revisão da LMP e talvez sua adequação.

\section{Conclusão}

Considerando-se os resultados obtidos, sugere-se uma existência de um padrão de prescrição na gestação que merece ser discutido visando à minimização de riscos efeitos adversos desnecessários, que pode comprometer os resultados do atendimento de pré-natal oferecido.

Recomenda-se, então, que medidas de intervenção sejam tomadas promovendo uma utilização racional dos medicamentos e dos recursos disponíveis, tais como: um programa de conscientização dos prescritores sobre a importância da prescrição por nome genérico, tanto em relação à questão legal, quanto em relação à otimização do seu trabalho e dos serviços de saúde que integra; discussão e, se necessário, revisão da LMP; divulgação da LMP existente; estímulo à educação continuada dos profissionais de saúde envolvidos com o prénatal, visando uma melhoria da qualidade das prescrições e conseqüentemente da atenção à gestante e, principalmente, discussões com todos os sujeitos do processo sobre a concepção de saúde-doença que norteia as práticas do serviço e da comunidade, visando resgatar a representação da gestação como um processo fisiológico normal, que exige cuidados, mas que não é patológico e, portanto, não implica necessariamente em intervenções curativas, entre elas a prescrição de medicamentos. 


\section{Resumo}

A utilização de medicamentos por gestantes deve ser considerada um problema de saúde pública, pois existem inúmeras lacunas sobre suas conseqüências ao feto e à gestante. Os estudos farmacoepidemiológicos podem contribuir para minimizar os riscos inerentes à terapia medicamentosa, traçando um perfil do consumo de medicamentos na gestação, propiciando uma avaliação do serviço e apontando medidas de intervenção. O objetivo deste trabalho foi traçar um perfil de prescrição de medicamentos em gestantes usuárias do SUS de Piracicaba, São Paulo, Brasil, utilizando-se os indicadores de prescrição recomendados pela Organização Mundial da Saúde e a classificação de medicamentos segundo risco ao feto do Food and Drug Administration. Encontrou-se que, na consulta de prénatal, 44,7\% das mulheres receberam prescrição medicamentosa, sendo o grupo de medicamento mais prescrito aquele que atua sobre o sistema hematopoiético $(34,9 \%)$. Do total, 26,0\% dos medicamentos foram incluídos na categoria $C$ de risco ao feto; $1,5 \%$ na categoria D e 1,5\% na categoria E. Estes dados sugerem uma medicalização da gestação e a necessidade de medidas de intervenção para uma utilização racional dos medicamentos no pré-natal.

Medicamentos; Mulheres Grávidas; Prescrição de Medicamentos

\section{Colaboradores}

T. A. Carmo escreveu o artigo e S. M. O. O. Nitrini contribuiu na revisão do manuscrito.

\section{Referências}

1. Mellin GW, Katzenstein M. The saga of Thalidomide (conclude): neuropathy to embryopathy, with case reports of congenital anomalies. N Eng J Med 1962; 267:1238-44.

2. Collaborative Group on Drug Use in Pregnancy. Medication during pregnancy: a intercontinental cooperative study. Int J Gynaecol Obstet 1992; 39:185-96.

3. Forfar JO, Nelson MM. Epidemiology of drugs taken by pregnant women: drugs that may affect the fetus adversely. Clin Pharmacol Ther 1990; 14:632-42.

4. Berglund F, Frodh H, Lundborg P, Prame B, Sannerstedt R. Drug use during pregnancy and breast-feeding. Acta Obstet Gynecol Scand Suppl 1984; 126:1-55

5. Fundo das Nações Unidas para a Infância. A prescrição: diretrizes para a utilização racional de medicamentos nos serviços básicos de saúde. São Paulo: Fundo das Nações Unidas para a Infância/ Universidade de São Paulo; 1992.

6. Meadows M. Pregnancy and the drug dilemma FDA Consumer [serial online] 2001; http://www. fda.gov.fdac/feature/2001/301-preg.html (acessado em16/Jan/2002).

7. Mengue SS, Schenkel EP, Duncan BB, Schmid MI. Uso de medicamentos por gestantes em seis cidades brasileiras. Rev Saúde Pública 2001; 35: 415-20.

8. Niebyl JR. Uso de drogas na gravidez. 2a Ed. São Paulo: Livraria Roca; 1989.

9. Fuchs FD, Wannmacher L. Farmacologia clínica: fundamentos da terapêutica racional. 2a Ed. Rio de Janeiro: Guanabara Koogan; 1998.

10. Boobis AR, Lewis P. Drugs in pregnancy: altered pharmacokinetics. Br J Hosp Med 1982; 28:56673.

11. Piper JM, Baum C, Kennedy DL. Prescription drug use before and during pregnancy in a Medicaid population. Am J Obstet Gynecol 1987; 157:148-56.

12. Fonseca MRCC. Uso de medicamentos na gravidez: uma abordagem farmacoepidemiológica [Dissertação de Mestrado]. Campinas: Universidade Estadual de Campinas; 1998.

13. Gomes KRO, Moron AF, Silva R, Siqueira AA Prevalência do uso de medicamentos na gravidez e relações com as características maternas. Rev Saúde Pública 1999; 33:246-54.

14. Barros JAC. Pensando o processo saúde doença: a que responde o modelo biomédico? Saúde Soc 2002; 11:67-84.

15. Castro CGSO. Estudos farmacoepidemiológicos: perspectiva para a racionalidade terapêutica pediátrica e perinatal. Boletim da Sobravime 1998; 30:13-5.

16. Strom BL. What is pharmacoepidemiology? In: Strom BL, editor. Pharmacoepidemiology. 2nd Ed. New York: John Wiley \& Sons; 1994. p. 3-11

17. Lee D, Bergman U. Studies of drug utilization. In Strom BL, editor. Pharmacoepidemiology. 2nd Ed. New York: John Wiley \& Sons; 1994. p. 379-93.

18. Hogerzeill HV. Promoting rational prescribing: an international perspective. Br J Clin Pharmacol 1995; 39:1-6. 
19. Organización Mundial de la Salud. Investigación del uso de los medicamentos. Boletín de Medicamentos Esenciales 1993; 14:1.

20. Rouquayrol MZ, Almeida Filho N. Epidemiologia \& saúde. 5a Ed. Rio de Janeiro: Medsi; 1999.

21. Organización Mundial de la Salud. Como investigar el uso de medicamentos en los serviços de salud. Geneva: Organización Mundial de la Salud; 1993.

22. Ministério da Saúde. Portaria no 1.179 de 17 de junho de 1996. Aprova as denominações comuns brasileiras, DCB. http:\|www.saude.gov.br (acessado em 16/Jan/2002).

23. Ministério da Saúde. Relação Nacional de Medicamentos Essenciais: RENAME - 2000. Brasília: Secretaria de Políticas de Saúde, Ministério da Saúde; 2000.

24. Organización Mundial de la Salud. Uso racional de los medicamentos. London: Organización Mundial de la Salud; 1986.

25. Chetley A. Medicamentos problema. 2a Ed. Lima: Acción Internacional para la Salud América Latina y el Caribe; 1995.

26. Lopes MCS, Ferreira LOC, Batista Filho M. Uso diário e semanal de sulfato ferroso no tratamento de anemia em mulheres no período reprodutivo. Cad Saúde Pública 1999; 15:799-808.

27. Freire WB. La anemia por deficiencia de hierro: estrategias de la OPS/OMS para combatila. Salud Publica Mex 1998; 40:199-205.

28. Zanini AC, Oga S. Guia de medicamentos/Grupo de Trabalho Zanini - Oga. 2a Ed. São Roque: IPEX Editora; 1997.
29. King PB, Lie RT, Irgens LM. Spina bifida and cleft lip among newborns of Norwegian women with epilepsy: changes related to the use of anticonvulsants. Am J Public Health 1996; 86:1454-6.

30. Werler MM, Hayes C, Lovik C, Shapiro S, Mitchell AA. Multivitamin supplementation and risk of birth defects. Am J Epidemiol 1999; 150:675-82.

31. Briggs GG, Freeman RK, Yaffe SJ. Drogas na gravidez e na lactação. 2a Ed. São Paulo: Livraria Roca Ltda; 1987.

32. BPR Guia de remédios. São Paulo: BPR - Consultoria, Projetos e Comércio Ltda; 1999.

33. School TO, Hediger ML, Bendich A, Schall JI, Smith WK, Krueger PM. Use of multivitamin mineral prenatal supplements influence on the outcome of pregnancy. Am J Epidemiol 1997; 146: $134-41$.

34. Diniz AS. Combate à deficiência de vitamina A: linhas de ação e perspectiva. Rev Bras Saúde Matern Infant 2002; 1:31-6.

35. Hogerzeil HV, Bimo, Ross-Degnan D, Laing RO, Ofori-Adjei D, Santoso B, et al. Field tests for rational drug use in twelve developing countries. Lancet 1993; 342:1408-10.

36. Santos V. Indicadores selecionados do uso de medicamentos - OMS, no município de Ribeirão Preto - SP [Tese de Doutorado]. São Paulo: Faculdade de Saúde Pública, Universidade de São Paulo; 1999

Recebido em 25/Abr/2003

Versão final reapresentada em 05/Jan/2004 Aprovado em 09/Mar/2004 